\title{
La estrategia aplicada a las universiddades
}

Fecha de recepción: 28 de junio de 2011 - Aceptación 29 de septiembre de 2011

\section{Luis Arturo Rivas Tovar - Adela Chávez - María del Pilar Peña}

\section{Resumen}

Este trabajo describe los modelos de gobierno universitarios más destacados en el mundo, haciendo énfasis en el caso de los gobiernos universitarios latinoamericanos donde se distinguen cuatro modelos de gobierno: el burocrático, el colegial, el empresarial y el híbrido. Este trabajo reflexiona sobre las estrategias que se pueden implementar en cada modelo, especialmente analizando y describiendo el caso mexicano.

\section{Abstract}

This work describes the models of university government more outstanding within the world, emphasizing on the case of the Latin American university governments where four types are distinguished: bureaucratic, school, entrepreneurial, and hybrid. This work reflects on the strategy of each type of model by analyzing and describing the Mexican case.

\section{Palabras clave}

Estrategia, gobierno universitario, México, gestión universitaria

\section{Keywords}

Strategy, University Government, Mexico, University Management

\section{Introducción}

El concepto de estrategia ha sido ampliamente trabajado en el mundo de la empresa pero en materia universitaria el tema ha sido abordado básicamente desde enfoques funcionales.

Dentro de las estrategias funcionales se destacan los trabajos de Didriksson (2005) y especialmente, La universidad de la innovación: una estrategia de transformación para la construcción de universidades del futuro; el de Salinas (2004) y Duart y Lupiáñez (2005) en su trabajo sobre La innovación docente y el uso de las nuevas tecnologías de la información y comunicación (TIC) en las universidades; el trabajo de Epper y Bates (2007) en su Estrategia para liderar el cambio tecnológico, los trabajos coordinados por (Sebastián 2003) sobre Estrategias de evaluación de profesores, la estrategia. (Galvis 1997) mediante el cual hace una orientación del rumbo que debe tomar la universidad de acuerdo al tipo de gobierno universitario, que escasamente se ha tratado. Igualmente es de destacar la obra de López (2000) con Las formas de gobierno y gobernabilidad institucional, 
mediante el cual destaca los trabajos de Kent (2007): La transparencia en las universidades y el derecho a la información, así como Reflexiones sobre la modernización irreflexiva y la claudicación de la pedagogía crítica.

El tema de la estrategia como orientación del rumbo que debe tomar la universidad de acuerdo al tipo de gobierno universitario se ha tratado escasamente. Es por ello que este trabajo busca reflexionar sobre esta laguna de conocimiento que rara vez suele ser descrita en artículos, ya que los investigadores educativos se han concentrado básicamente en las estrategias funcionales (Galvis 1997).

El planteamiento de este ensayo, se centra en buscar una respuesta entre las estrategias de administración educativa del mundo universitario y los modelos de gobierno universitario más destacados, pues se trata de una investigación documentada que se apoya en la lectura de las fuentes citadas en la bibliografía y la observación participante de los autores, producto de sus experiencias profesionales.

\section{Reseña de autores Luis Arturo Rivas Tovar (México) Instituto Politécnico Nacional (IPN) larivas33@hotmail.com \\ Catedrático ESCA México. Coordinador de la Maestría en Administración Pública}

Adela Chávez (México)

Instituto Politécnico Nacional (IPN)

adelita1122@yahoo.com.mx

Catedrática ESCA IPN

María del Pilar Peña (México) Instituto Politécnico Nacional penacruz@hotmail.com

Doctora e investigadora en el Instituto Politécnico Nacional. Directora de la Revista. Investigación Administrativa. Premio de la Presidencia de la República y Medalla Ignacio Altamirano.

\section{MARCO CONTEXTUAL DE LAS INSTITUCIONES UNIVERSITARIAS}

En México existen 1396 Instituciones de educación superior (IES), de acuerdo a datos de (ANUIES, 2008).

La heterogeneidad es una de las características de las universidades iberoamericanas. En los últimos veinte años y como consecuencia de las políticas públicas en educación superior de la Unión Europea y de la propia evolución de las universidades, se ha producido una cierta homogeneización en las universidades españolas y portuguesas.

Las universidades latinoamericanas son muy diversas y su heterogeneidad ha aumentado a la par del enorme crecimiento que ha tenido la población estudiantil de tercer nivel en la región (Yarzábal, 1997). Los indicadores de dicha heterogeneidad son variados y entre ellos se destacan los siguientes:

1. Sostenibilidad institucional

2. Perfil académico y posicionamiento

3. Experiencia de aprendizaje de los estudiantes

4. Investigación

5. Transferencia de conocimientos y relaciones

6. Salud financiera

7. Inmuebles e infraestructura

8. Desarrollo de recursos humanos y del personal

9. Gobernabilidad, dirección y gestión

10. Proyectos institucionales (acreditación)

11. Ranking mundial en la web.

En América Latina existen entre cinco mil y seis mil instituciones de educación superior, de las que aproximadamente el $15 \%$ son universidades, es decir, sólo exis- 
ten unas 900 universidades. A mediados de la década de 1990 había 297 universidades públicas y 380 privadas. Es decir en un periodo de veinte años se han sumado más de 200 universidades. Las universidades públicas, el $68 \%$, fueron creadas a partir de 1950 y las universidades privadas, el porcentaje de crecimiento es aún más marcado, ya que el 96\% se creó a partir de la década de los 50.

Esto supone que el mundo universitario tanto en América Latina como en el mundo ha dejado de ser un espacio reservado a lo público y la iniciativa privada con ánimo de lucro o sin él, cada vez tiene mayor relevancia tanto en la cobertura como en el prestigio de sus universidades.

El tema de la estrategia en universidades está asociado al tipo de gobierno que se tiene, ya que dependiendo de este tipo de gobierno y de los fines e intereses que persiga, una universidad puede tener distintos tipos de estrategia.

Por la importancia que tuvo la Conferencia de Rectores de las universidades españolas del año 2000, se tomaron en cuenta algunos resultados obtenidos que aún prevalecen en la actualidad, ya que como se estableció en dicha reunión:

"Junto a las asociaciones que forman las propias universidades o miembros de la comunidad, también cabe mencionar a los organismos e instituciones que a nivel local, autonómico o internacional, velan por los intereses y el buen funcionamiento de la educación superior."

Como referencia, para el caso de México, en la gráfica 1 se presenta la población escolar de educación superior a nivel nacional para el ciclo escolar 2007 a 2008.

\section{Gráfica 1 Población escolar de educación superior 2007-2008}

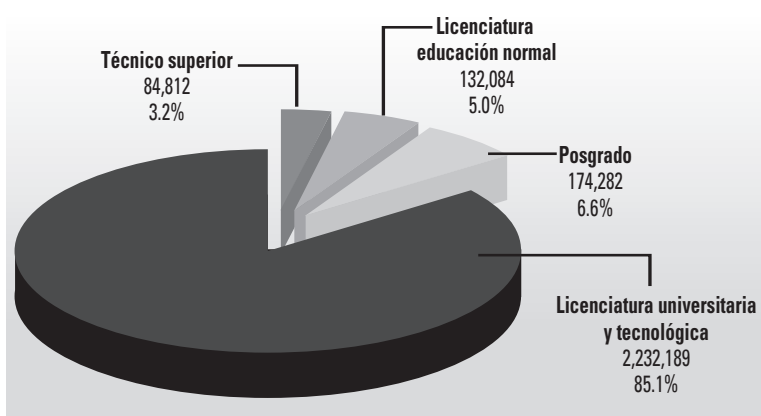

Fuente: elaboración propia con datos de los Formatos 911 9A y 911 9B, ciclo escolar 2007 - 2008

En la gráfica 2 se menciona la población escolar por régimen para el mismo periodo. Como puede apreciarse la preeminencia del sector público en México resulta indiscutible, ya que supera al sector privado en todos los órdenes desde la licenciatura, el posgrado hasta los técnicos superiores.

\section{Gráfica 2. Población escolar de educación superior por régimen 2007-2008}

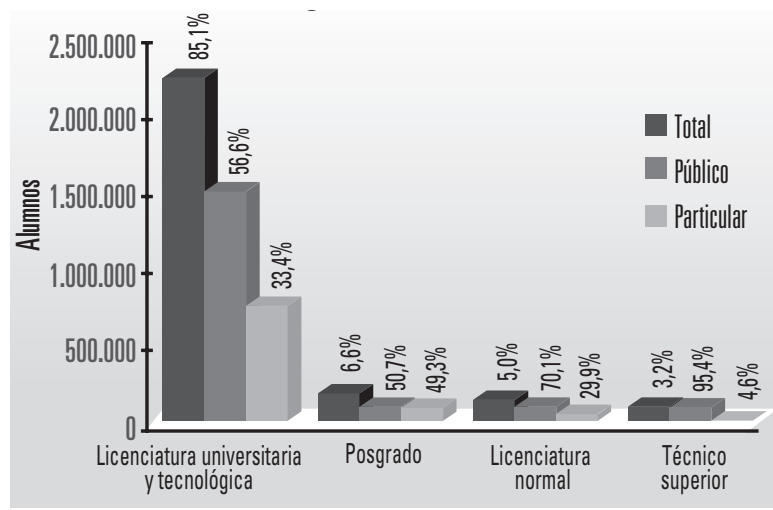

Fuente: elaboración propia con datos de los Formatos 911 9A y 911 9B, ciclo escolar 2007 - 2008

\section{MODELOS DE GOBIERNO UNIVERSITARIO}

En diversas partes del mundo, existen cuatro modelos de gobierno universitario y en consecuencia, cuatro estrategias universitarias (ver cuadro 1 ). 


\section{Cuadro 1. Modelos de gobierno universitario y de estrategia}

\begin{tabular}{|l|l|}
\hline 1 & $\begin{array}{l}\text { Europeo Continental: equilibrio entre el } \\
\text { poder de los profesores y el poder de la } \\
\text { burocracia estatal. }\end{array}$ \\
\hline 2 & $\begin{array}{l}\text { Británico: predominio del poder de los } \\
\text { profesores y modesta influencia de } \\
\text { comités de supervisión y administra- } \\
\text { dores. }\end{array}$ \\
\hline 3 & $\begin{array}{l}\text { Estadounidense: bajo poder de profe- } \\
\text { sores y gran preponderancia del poder } \\
\text { de comités de supervisión y adminis- } \\
\text { tradores }\end{array}$ \\
\hline 4 & $\begin{array}{l}\text { Latinoamericano: varios modelos: } \\
\text { Burocrático, Colegial y Empresarial. }\end{array}$ \\
\hline
\end{tabular}

Fuente: elaboración propia.

De los cuatro modelos de gobierno mencionados la investigación se concentra en el latinoamericano que si bien no es un modelo puro, dado que tiene características de los otros tres modelos, existe la complejidad adicional que cambian por país y región. Estos pueden ser resumidos en cuatro tipos que son los expuestos en la gráfica 3 .

\section{Gráfica 3. Modelos de gobierno universitario en América Latina}

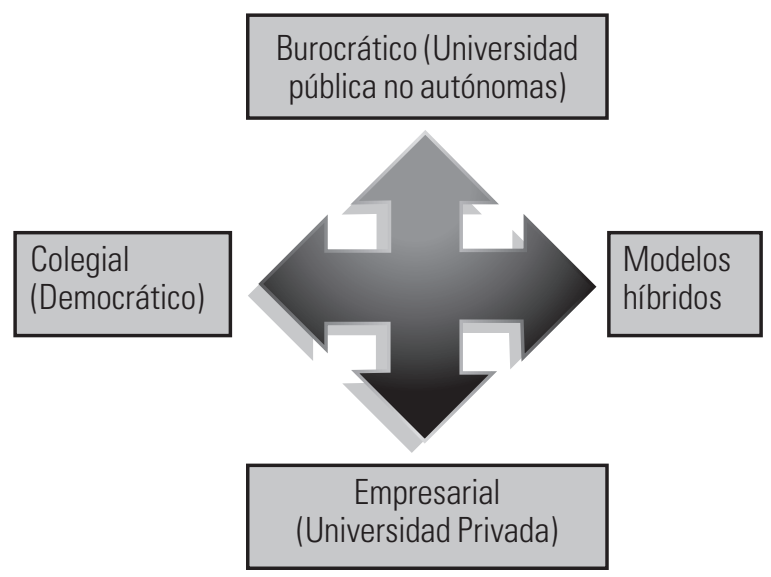

Fuente: elaboración propia.

\section{Modelos de gobierno en América Latina}

a) El modelo burocrático (universidad pública no autónoma)

Este modelo es propio de las universidades públicas no autónomas, pues el poder de los que proveen el presupuesto a la universidad, les permite incidir en el nombramiento de sus principales directivos. El nombramiento del rector se considera una facultad indiscutible del gobernador o del presidente de la República quien de esta manera controla la universidad, la cual es tradicionalmente fuente de disidencia organizada porque es mirada con preocupación por el grupo que detenta el poder político y económico.

La administración pública asume la responsabilidad de financiar y controlar el funcionamiento y el gasto de la universidad, por ello una de las grandes tareas del rector y de su cuerpo de gobierno es conciliar intereses entre los actores que proponen el presupuesto anual y los responsables de ejercerlo, de una manera clara y honesta. Esto suele ser tarea del gobernador o el presidente y en casos de alta competencia política en el poder legislativo en las comisiones de educación de las cámaras de diputados y senadores.

En compensación de esta dependencia del dinero público, el poder ejecutivo suele nombrar o ejercer una influencia determinante en la designación de los principales responsables de la gestión universitaria, ejerciendo frecuentemente una estricta supervisión de su actuación.

La administración del Estado por vía del Ministerio de Educación expide y garantiza la validez académica y profesional de los títulos otorgados por la universidad y define sus contenidos.

En este modelo, la universidad se concibe fundamentalmente como un organismo de 
la administración educativa, aunque tenga un cierto grado de autonomía o especificidad administrativa.

En consonancia a esta tradición y control, la organización interna de la universidad es de corte tradicional basada en el modelo de vice rectorías o secretarias académicas, divisiones y departamentos. Es una universidad vertical centralizada la cual controla a su vez a las escuelas o facultades a través de la autorización presupuestal y el nombramiento de sus directores.

El personal directivo de la universidad tiene el carácter de funcionario público y su proceso de selección y de promoción está fuertemente reglamentado por la administración educativa aun cuando estén sindicalizados sus profesores.

La gestión de la universidad se rige por las normas de la Administración Pública (derecho administrativo, intervención financiera, inspección de servicios, etc.). Suelen existir órganos internos de control cuya tarea es recordar al rector y a su equipo, el cumplimento de una multitud de normas y reglamentos que suelen con frecuencia demorar la capacidad de renovación y cambio estratégico.

Aunque existen órganos colegiados de gobierno en la universidad, estos tienen un carácter consultivo o de propuesta, complementado frecuentemente con la cesión de competencias en cuestiones menores, ligadas a intereses corporativos de los miembros de tales órganos (carreras profesionales, traslados, reconocimiento de méritos, etc.). Sin embargo, las grandes decisiones sobre la universidad no se toman en estos órganos, sino en la cúspide del poder ejecutivo.

\section{Ib) El modelo colegial (universidades autónomas)}

En este modelo el financiamiento de las universidades, en su mayor parte, es de carácter público, pero la administración pública apenas interviene en la gestión interna de la institución. Con frecuencia ocupan una silla en el consejo universitario sin embargo, la elección corresponde a miembros notables de estos cuerpos de gobierno o bien a un sistema de votación ponderados que reflejan la importancia de los grupos de interés de la universidad.

Los órganos colegiados ejercen directamente el gobierno de la institución o eligen libremente a los responsables del mismo.

La universidad decide el contenido de sus programas de enseñanza, garantiza la validez académica de sus títulos y negocia con los organismos públicos y asociaciones profesionales su reconocimiento para el ejercicio profesional.

La gestión de la universidad se rige por normas propias basadas en las tradiciones académicas y en el contrapeso de poderes de los distintos sectores académicos.

Los órganos colectivos del gobierno de la institución no son sólo consultivos, sino que también pueden elegir a los órganos personales, tomar decisiones ejecutivas y realizar funciones de control.

La organización interna es de carácter tradicional, basada en las disciplinas académicas, y con poca dependencia de los requerimientos del mercado profesional. Los profesores son contratados libremente por la propia universidad, mediante comisiones de pares que actúan con criterios propios, de carácter académico. Este modelo es democrático y el conflicto estalla de vez en cuando sobre todo en los procesos de negociación del contrato colectivo y las
Lagestión de la universidad se rige por las normas de la Administración Pública (derecho administrativo, intervención financiera, inspección de servicios, etc.). Suelen existir órganos internos de control cuya tarea es recordar al rector y a su equipo, el cumplimento de una multitud de normas $y$ reglamentos que suelen con frecuencia demorar la capacidad de renovación y cambio estratégico. 
revisiones salariales. Por regla general los sindicatos tienen compromiso ideológico y político que los unen con otros actores lo cual hace potencialmente grave el estallido de un conflicto.

\section{c) Modelo empresarial (universidad privada)}

La financiación es predominantemente privada ( sin ánimo de lucro o con fines lucrativos ) y la gestión se lleva a cabo a través de órganos equivalentes a un consejo de administración que está constituido de acuerdo con las normas establecidas por el titular de la institución. Este consejo de administración u órgano equivalente designa o contrata a los responsables máximos del gobierno de la universidad.

Cada universidad decide el contenido de sus cursos, garantiza la validez de sus títulos y negocia su reconocimiento con la administración, con otras universidades y con asociaciones profesionales a través de sistemas de acreditación y de certificación de la calidad de las mismas o mediante procedimientos equivalentes.

La dirección de la universidad contrata libremente a sus profesores, aunque para ello se apoye en un asesoramiento académico tanto interno como externo.

Cada universidad constituye una entidad privada cuya gestión se lleva cabo con criterios de gerencia profesional. Por tanto, en este contexto, los órganos colectivos académicos son meramente consultivos y carecen de funciones propiamente de gestión y de planificación económica.

La estructura interna de la organización es de carácter matricial y flexible, con gran capacidad de adaptación a los cambios del entorno social y científico. En este modelo todos los profesores como los directivos son empleados, por lo general las universidades carecen de sindicatos o bien estos tienen un poder muy limitado. Las grandes decisiones sobre la universidad no suelen ser consultadas con los colegios que tienen un rol solo en la parte académica y no en la estrategia general.

El objetivo central es el crecimiento de la matrícula y los beneficios. Si bien el beneficio social siempre está implícito, no es usual que se de un beneficio económico. El modelo privado suele hacer énfasis y tener preeminencia en el campo de las ciencias sociales.

\section{d) Modelo híbrido}

Es un modelo poco común, en América Latina se identifica, entre algunos casos de órganos desconcentrados, el mejor ejemplo en el Instituto Politécnico Nacional de México, la desconcentración es un acto de legislación por medio del cual se transfieren ciertas facultades de un órgano central a los organismos que forman parte de su propia estructura, con la finalidad de que la actividad que realiza la administración se haga de un modo pronto y expedito.

Destaca, Espinoza (1986), que "el organismo desconcentrado tiene cierta autonomía a la que se le llama técnica, que significa el otorgamiento de facultades de decisión limitadas y cierta autonomía financiera presupuestaria".

No obstante el otorgamiento que la ley hace de dicha autonomía técnica y presupuestaria, el organismo carece de capacidad jurídica.

El organismo administrativo además carece de patrimonio propio por lo que no formula su propio presupuesto, sino 
que este le es determinado y asignado por el titular de la entidad central de la que depende.

Respecto a las facultades administrativas que son otorgadas al organismo administrativo, estas pueden ser, en todo momento, desempeñadas directamente por el órgano central del cual depende, por lo que no existe una verdadera transferencia de facultades.

\section{IV.CLASIFICACIÓN DE MODELOS DEAMÉRICA LATINA}

El cuadro 2 permite clasificar los modelos de gobierno universitario en América Latina en función de las variables, a saber: financiación, directivos, programa académico, titulaciones, profesores, estatuto jurídico, modelo de gestión, órganos colegiados y organización, por lo tanto es posible hacer y describir para una mejor comprensión del lector, la siguiente clasificación:

Cuadro 2. Clasificación de modelos de gobierno universitario

\begin{tabular}{|l|l|l|l|}
\hline VARIABLE & BUROCRÁTICO & COLEGIAL & EMPRESARIAL \\
\hline Financiación & Sólo pública & $\begin{array}{l}\text { Pública o privada no } \\
\text { luvrativa }\end{array}$ & Privada lucrativa o no \\
\hline Directivos & Nombrados & Electos & Contratados \\
\hline Programa académico & Definido por el estado & $\begin{array}{l}\text { Definido por la } \\
\text { academia }\end{array}$ & Según demanda \\
\hline Titulaciones & $\begin{array}{l}\text { Emitidos por } \\
\text { ministerio }\end{array}$ & $\begin{array}{l}\text { Emitidos por la } \\
\text { universidad }\end{array}$ & $\begin{array}{l}\text { Acreditación nacional } \\
\text { internacional }\end{array}$ \\
\hline Profesores & $\begin{array}{l}\text { Funcionarios del } \\
\text { estado }\end{array}$ & Contrato académico & Contrato laboral \\
\hline Estatuto jurídico & $\begin{array}{l}\text { Organismo del estado } \\
\text { (descentralizado) }\end{array}$ & $\begin{array}{l}\text { Organización } \\
\text { autónoma }\end{array}$ & $\begin{array}{l}\text { Empresa privada o } \\
\text { asociación civil }\end{array}$ \\
\hline Organos colegiados & $\begin{array}{l}\text { Consultivos y } \\
\text { electivos }\end{array}$ & Ejecutivos y lectivos & Sólo consultivos \\
\hline Organización & Burocracia mecánica & $\begin{array}{l}\text { Burocracia } \\
\text { profesional }\end{array}$ & $\begin{array}{l}\text { Adhocracia, } \\
\text { horizontal, celular o } \\
\text { virtual }\end{array}$ \\
\hline
\end{tabular}

Fuente: Elaboración propia adaptado de Bricall, (2000).

\section{V.MODELOS DE GOBIERNO UNIVERSITARIO EN MÉXICO}

1) Los modelos de gobierno universitario más destacados

En México uno de los modelos de gobierno universitario más destacados, se ubica en el colegial y el empresarial, debido a que responde a las características mencionadas en el cuadro 2, en el modelo colegial se destaca la UNAM
(Universidad Autónoma de México y la UAM (Universidad Metropolitana), mientras que en el modelo empresarial se destaca el ITESM (Instituto Tecnológico de Estudios Superiores de Monterrey). Entre el modelo burocrático cabe mencionar al IPN (Instituto Politécnico Nacional) y a algunas de las universidades estatales que no son autónomas. 
El tratar de relacionar los modelos a distintos tipos de universidad, resulta complicado por la gran diversidad de IES que existen en México. Haciendo un esfuerzo por resumir podríamos decir que son ocho tipos:

1. Universidades públicas estatales (43)

2. Universidades privadas (773)

3. Institutos tecnológicos estatales (108)

4. Institutos tecnológicos federales (110)

5. Universidades interculturales (5)

6. Escuelas normales superiores (273),

7. Universidades politécnicas (23) y

8. Universidades tecnológicas (61)

Lo que hace un gran total de 1396 universidades. A continuación se describen brevemente cada una de ellas.

\subsection{Universidades públicas estatales}

Estas instituciones estatales desarrollan las funciones de docencia, generación y aplicación innovadora del conocimiento, así como de extensión y difusión de la cultura, y suman 43 universidades. Aunque la mayoría son autónomas, el gobernador tiene una alta influencia en el nombramiento del rector.

\subsection{Universidades privadas}

Su estructura se conforma por 773 universidades con posgrado, 120 de ellas con doctorado, y se dividen en dos grandes grupos:

a) Universidades de muy distintos tipos y tamaños (algunas de las cuales tienen diversos planteles), dedicadas a la formación en diversos campos y, en algunos casos, en todos los niveles. Entre todas ellas, cubren una matrícula del 33\% del total del público universitario. Para ilustrar la diversidad de estas instituciones, Se ha de explicar que por un lado, se encuentran instituciones con una matrícula elevada, con programas reconocidos de licenciatura, maestría y doctorado en un número importante de campos del conocimiento, pero en el otro extremo se encuentra un contingente muy grande de instituciones pequeñas, a veces con matrículas inferiores a los 100 alumnos, que ofrecen un número muy limitado de programas de licenciatura, generalmente vinculados con el área económico-administrativa.

b) Escuelas normales, dedicadas principalmente a la formación de maestros de educación básica. El conjunto de escuelas normales privadas atendió cerca de $55 \mathrm{mil}$ alumnos, lo que representa un poco más del $2 \%$ de la matrícula total de nivel superior y el $37 \%$ de la matrícula atendida por las instituciones que preparan a los profesionales de la educación básica. (Subsecretaria de Educación Superior, Secretaria de Educación Pública de México 2010).

\subsection{Institutos tecnológicos}

El Sistema Nacional de Institutos Tecnológicos (SNIT) tiene como prioridad formar profesionales competentes y propiciar el desarrollo nacional mediante planes y programas de estudio pertinentes para la realidad de cada región. El SNIT está conformado por 218 instituciones en los 31 estados de la República, clasificados en 108 Institutos Tecnológicos estatales y 110 Institutos Tecnológicos Federales. (Secretaria de Educación Pública, México 2010).

\subsection{Universidades interculturales}

Las Universidades Interculturales tienen como objetivo impartir programas formativos en los niveles: profesional aso- 
ciado, licenciatura, especialización, maestría y doctorado, pertinentes al desarrollo regional, estatal y nacional, orientados a formar profesionales comprometidos con el desarrollo económico, social y cultural en los ámbitos comunitario, regional y nacional, cuyas actividades contribuyan a promover un proceso de valoración y revitalización de las lenguas y culturas originarias.

Los datos de la población estudiantil en las universidades interculturales no son precisos a la fecha.

\subsection{Educación normal superior}

Hay 273 escuelas normales públicas en el país. La matrícula de estas escuelas está compuesta por más de 93 mil estudiantes. (Secretaria Educación Pública, de: http:// ses4.sep.gob.mx/wb/ses/federales).

\subsection{Universidades Politécnicas}

Las Universidades Politécnicas son un conjunto de instituciones públicas comprometidas con el desarrollo económico y social de la nación, con proyección internacional, constituyéndose en factor clave para el progreso de los 23 estados de la república donde se localizan.

\subsection{Universiddades tecnológicas}

Las Universidades Tecnológicas (UTs) ofrecen a los estudiantes que terminan la educación media superior, una formación intensiva que les permite incorporarse en corto tiempo (luego de dos años), al trabajo productivo o continuar estudios en el nivel de licenciatura en otras instituciones de educación superior. El Modelo Educativo de las UTs está orientado al aprendizaje como un proceso a lo largo de la vida. Actualmente hay 61 Universidades
Tecnológicas en 26 estados de la República. El que estudia en estas instituciones obtiene el título de Técnico Superior Universitario.

\section{Universidades preferidas de México}

El grupo editorial Reader's Digest diseñó una metodología basada en una encuesta que se aplicó a un conjunto de 780 gerentes y directores de recursos humanos, 475 profesores de educación superior tanto en instituciones públicas y privadas, y a 475 alumnos que cursan carreras en todas las áreas del conocimiento.

La idea de esta empresa es definir una clasificación de las mejores universidades que, si bien puede ser cuestionable, es una de las pocas clasificaciones que existen sobre calidad.

El cuadro 4, resume las primeras diez posiciones.

\section{Cuadro 4. Las 10 universidades preferidas públicas y privadas de México}

\begin{tabular}{|l|l|l|}
\hline Lugar & Universidad & Status \\
\hline 1 & UNAM & Pública \\
\hline 2 & ITESM & Privada \\
\hline 3 & IPN & Pública \\
\hline 4 & Universidad Iberoamericana & Privada \\
\hline 5 & Universidad del Valle de México & Privada \\
\hline 6 & Instituto Tecnológico Autónomo de México & Privada \\
\hline 7 & Universidad Anáhuac & Privada \\
\hline 8 & Universidad TecMilenio & Privada \\
\hline 9 & Universidad de Guadalajara. & Pública \\
\hline 10 & Universidad Autónoma Metropolitana & Pública \\
\hline
\end{tabular}

Fuente: adaptación con base en Guía Universitaria de Readers Digest 2011 
Es importante anotar de la información anterior, que las IES públicas mantienen un equilibrio sobre la preferencia del $50 \%$; por otro lado, existen cinco universidades públicas dentro de las diez más solicitadas en el país y de las tres IES que encabezan la lista, solo una es privada.

\section{La estrategia en el mundo universitario mexicano}

El cuadro 5 presenta las diversas estrategias que pueden seguir las Universidades, en función de sus objetivos y de su tipo de gobierno.

Dentro de estas estratégicas se distinguen tres grandes grupos de estrategias por su alcance: a) estrategias corporativas o macro estrategia, b) estrategias de unidades de negocios y c) estrategias funcionales.
Dentro de las estrategias corporativas o macro estrategias cabe mencionar las estrategias de crecimiento. Estas últimas ocurren en una universidad por diversos motivos. Es común en universidades privadas exitosas que deciden emprender el crecimiento en otras ciudades, o universidades burocráticas o colegiales que deciden ampliar sus sedes cuando sus presupuestos lo permiten. Más comunes son las estrategias de segmentos, son propias de universidades que deciden escalar a un nivel de conocimiento superior, por ejemplo aquellas que abren una sección de posgrado o que teniéndola deciden ofrecer los estudios de doctorado. Cada estrategia de crecimiento supone un análisis de recurso y capacidad para evaluar si existen los elementos indispensables para realizar con éxito el crecimiento anhelado. (Ver cuadro 5).

\section{Cuadro 5. Estrategias aplicadas a las universidades}

\begin{tabular}{|c|c|c|c|}
\hline \multirow{6}{*}{$\begin{array}{l}\text { Estrategias corporativas } \\
\text { Tienen objetivos de crecimiento, } \\
\text { estabilidad y supervivencia. Exigen } \\
\text { la búsqueda de la mejor inversión } \\
\text { de recursos y negocios para lograrlo }\end{array}$} & \multirow[t]{2}{*}{ Estrategias de crecimiento } & $\begin{array}{l}\text { Estrategias de } \\
\text { crecimiento interno }\end{array}$ & $\begin{array}{l}\text { - Crecimiento del } \\
\text { - segmento de mercado } \\
\text { - Nuevos productos } \\
\text { - Nuevos mercados } \\
\text { - Diversificación } \\
\text { - Integración vertival }\end{array}$ \\
\hline & & $\begin{array}{l}\text { Estrategias de } \\
\text { crecimiento externo }\end{array}$ & $\begin{array}{l}\text { - Adquisiciones } \\
\text { - Fusiones } \\
\text { - Alianzas estratégicas } \\
\text { - Franquicias }\end{array}$ \\
\hline & Estrategias de estabilidad & \multicolumn{2}{|c|}{$\begin{array}{l}\text { - Saneamiento (turnaround) } \\
\text { - Reconversión }\end{array}$} \\
\hline & Estrategias de asociativas & \multicolumn{2}{|l|}{$\begin{array}{l}\text { - Redes } \\
\text { - Clústers }\end{array}$} \\
\hline & Estrategias de supervivencia & \multicolumn{2}{|l|}{$\begin{array}{l}\text { - Desinversión } \\
\text { - Liquidación }\end{array}$} \\
\hline & Estrategias de internacionalización & \multicolumn{2}{|l|}{$\begin{array}{l}\text { - Inversión directa } \\
\text { - Join venture }\end{array}$} \\
\hline
\end{tabular}




\begin{tabular}{|l|l|}
\hline \multirow{2}{*}{$\begin{array}{l}\text { Estrategias de unidades de } \\
\text { negocios }\end{array}$} & Estrategias de diferenciación \\
\cline { 2 - 2 } Su objetivo es competir & Estrategias de liderazgo en costos \\
\cline { 2 - 2 } & Estrategias de enfoque o nicho \\
\hline \multirow{4}{*}{$\begin{array}{l}\text { Estrategias funcionales } \\
\text { Armonizan los recursos de cada }\end{array}$} & Estrategias de mercadotecnia \\
\cline { 2 - 2 } área a la estrategia global & Estrategias de producción \\
\cline { 2 - 2 } & Estrategias de recursos humanos \\
\cline { 2 - 2 } & Estrategias financieras \\
\cline { 2 - 2 } & Estrategias tecnológicas (I\&D) \\
\hline
\end{tabular}

Fuente: elaboración propia.

Las estrategias de crecimiento externo, resultado de fusiones, adquisiciones y alianzas estratégicas son más raras. Se dan básicamente en universidades privadas sobre todo en el caso de fusiones y adquisiciones. La compra de Silvan International de la Universidad del Valle de México es un caso reciente.

En el caso de alianzas estratégicas, se puede observar que si bien es común la aplicación de estas estrategias en el ámbito privado, los convenios de doble titulación y los de intercambio de profesores y alumnos son también algunos casos.

Resulta poco frecuente ver la estrategia de franquicias en el gobierno universitario, pues en el mundo universitario mexicano es un modelo poco explorado.

Las estrategias de estabilidad se distinguen en a) estrategias de saneamiento, b) reconversión y c) reingeniería, las cuales se observan más en universidades privadas. Aunque en las universidades públicas se hace una revisión de prácticas cada vez que hay cambio de autoridades, no se podría afirmar que estos ejercicios administrativos son estrategias de saneamiento y reconversión.

Las estrategias de supervivencia son más extrañas aún porque raramente desaparece una universidad. En un mercado de jóvenes con bajas tasas de escolaridad universitaria, abrir una nueva universidad resulta un magnifico negocio que siempre tiene tasas ascendente por las dinámicas demográficas.

Las estrategias de internacionalización que también son raras en el mundo universitario mexicano, salvo el caso del Tecnológico de Monterrey, se ha atrevido a hacer expansiones a Centro y Suramérica, es de resaltar el caso del Silvan International. Por la poca frecuencia de esta estrategia, las universidades tanto públicas como privadas tienen un enfoque doméstico.

En materia de estratégicas de asociatividad, se observan acuerdos entre universidad y empresa sobre todo en el caso de universidades privadas donde las universidades firman convenios donde se comprometen a dotar a la industria de trabajadores altamente calificados que ayuden a la construcción de la infraestructura o para que envíe a sus ejecutivos o a sus empleados a programas de formación continua.

En cambio, las estrategias de negocios en el mundo universitario se encuentran ampliamente exploradas. Se observa más en universidades privadas, por ejemplo, el Tecnológico de Monterrey ha desarrollado técnicas para orientar sus servicios 
a un mercado hacia la clase media alta, con sus diversos campus. La universidad Anáhuac está orientada a las clases altas, sirviendo a colectivos muy importantes e influyentes como son las comunidades judías y libanesas. Hay un importante segmento de universidades privadas de orientación religiosa enfocadas en segmentos medio- alto y alto.

Las universidades públicas por lo general se orientan a segmentos de clase media y segmentos de origen rural. Se presenta el caso de escuelas estatales que, a pesar de tener precios bajos son la mejor opción en calidad sobre todo a niveles de posgrado e investigación donde casi todas las universidades privadas suelen ser pocos atractivas y competitivas. Escasamente tienen investigadores nacionales y sus claustros rara vez son conformados por doctores de tiempo completo.

Salvo el caso del Tecnológico de Monterrey casi todas las universidades privadas, con excepción de las salvedades mencionadas, tienen una estrategia de posicionamiento orientado a las clases medias, las cuales son rechazadas de las grandes escuelas públicas federales.

Finalmente las estrategias funcionales se observan en casi todas las universidades y podría decirse que son las estrategias más comúnmente aplicadas. Mejoras en la calidad, en la certificación de sus claustros en la adquisición e integración de su sistema de información, en la adquisición de nuevas formas de educación virtual como el e-learning, entre otras, son comunes en las agendas estratégicas.

El concepto de estrategia en el mundo universitario es casi tan refinado y diverso como en todas las organizaciones; sin embargo, está pobremente documentado, especialmente a nivel de estrategias corporativas.

Es una tarea y un reto de interés el documentar los distintos proyectos estratégicos de las universidades latinoamericanas.

\section{VI.CONCLUSIONES}

- En México, los modelos de gobierno universitario más destacados se ubican en el colegial y en el empresarial. En el colegial se destaca la UNAM (Universidad Autónoma de México) y la UAM (Universidad Metropolitana), mientras que en el empresarial se destaca el ITESM (Instituto Tecnológico de Estudios Superiores de Monterrey).

- El mundo universitario, tanto en América Latina como en otras partes del mundo, ha dejado de ser un espacio reservado a lo público y la iniciativa privada con ánimo de lucro o sin él, cada vez tiene mayor relevancia tanto en la cobertura como en el prestigio de sus universidades.

- Los modelos de gobierno universitario en América Latina son:

1. Burocrático (Universidad pública no autónoma)

2. Colegial (democrático)

3. Empresarial (Universidad Privada)

4. Modelos híbridos (Universidad como órgano desconcentrado de la administración pública, caso IPN).

El tema de la estrategia en las universidades está asociado al tipo de gobierno que adapta, ya que dependiendo del tipo de gobierno y de los fines que persiga, una universidad puede tener distintos estilos de estrategia bien sean corporativas, de negocios y/o funcionales. 


\section{Bibliografía}

1. ANUIES (2008), recuperado 19 de junio de 2011 de: http://www.anuies.mx/ servicios/e_educacion/index2.php

2. Bricall, J. M. (2000), Conferencia de Rectores de las universidades españolas (CRUE)

3. Barcelona: Informe Universidad.

4. CONACYT (2006), Indicadores básicos de ciencia y tecnología, recuperado 17 de enero de 2011 de www.conacyt.mx.

5. Didriksson A. (2005), La Universidad de la innovación: una estrategia de transformación para la construcción de universidades del futuro, México: Plaza y Valdez.

6. Duart, J. (2005), Monográfico Calidad y uso de las TIC en la universidad: estrategia y transformación. Revista de Universidad y Sociedad del Conocimiento (RUSC). [Artículo en línea]. UOC. Vol. 2, No 1, recuperado el 22 de octubre de 2010: en http:// rusc.uoc.edu/ojs/index.php/rusc/

7. Duart, J. y Lupiáñez L. (2011), Estrategias en la introducción y uso de la perspectiva organizativa del e-learning. Introducción, recuperado el 11 de octubre de 2010 en: www.raco.cat/index.php/DIM/article/view/87135/112211

8. Duart, L., Sangrà, A. (2000), Formación universitaria por medio de la web: un modelo integrador para el aprendizaje superior. (comps.) Aprender en la virtualidad. Barcelona: Gedisa.

9. Epper, R., Bates, A. (2004), Enseñar al profesorado cómo utilizar la tecnología. Barcelona: UOC.

10. Espinoza, B. M., (1986), Lineamientos de Derecho Público Mexicano, p.141. Primera edición. Tijuana: Cárdenas Editores.

11. Galvis, A. (2007). Pensamiento Estratégico: Manera Proactiva de asumir los retos de una organización. Adaptado de Galvis (1997), Capítulo 2 de Usos estratégicos de informática. Madrid: Gedisa.

12. Kent, R. S. (2007). La transparencia en las universidades: El derecho a la información, la modernización irreflexiva y la claudicación de la pedagogía crítica. CPU-e, Revista de Investigación Educativa, 4. Recuperado el 20 de junio de 2011, de http://www.uv.mx/ cpue/num4/critica/kent_transparencia_derecho.htm

13. López, Z. R. (1988), Una aproximación a las formas de gobierno de las universidades públicas en sociología, vol. 13, (36), enero abril 1988, pp. 221-251.

14. Lopez, Z. R. (2001). Las formas de gobierno de las IES mexicanas en Revista de la Educación Superior, Vol. 30, num. 118, Mèxico, Anuies, pp. 55-77 Índice de Revistas de Educación Superior e Investigación Educativa (IRESIE), Recuperado el 15 abril 2011, de: http://132.248.192.201/seccion/bd_iresie/iresie_busqueda. php?indice $=$ autor\&busqueda $=$ LOPEZ\%20ZARATE,\%20ROMUALDO\&par $=\& a_{-}$ inicial=\&a_final $=\&$ sesion

15. Secretaria de educación pública (2010) recuperado de Educación superior publica http://www.ses4.sep.gob.mx

16. Salinas, J. (2004). Innovación docente y uso de las TIC en la enseñanza universitaria. En Revista de Universidad y Sociedad del Conocimiento (RUSC). Artículo en línea, pp.1-16 
17. UOC., vol.1 (1), recuperado el 22 enero de 2010, de: http://www.uoc.edu/rusc/dt/esp/ salinas1104.pdf>

18. Sangrá, A. y Gonzáles, M. (2004). La transformación de las universidades a través de las TIC: discursos y prácticas. Barcelona: UOC

19. Sebastián J. (2003). Estrategias de cooperación universitaria para la formación de investigadores en Iberoamérica organización de estados Iberoamericanos. Madrid: OEI.

20. Selecciones del Reader Digest, recuperado el 30 de abril de 2011, de: http://www.selecciones.com/guia/top_ten.html

21. Subsecretaria de educación superior pública, Institutos Tecnológicos, recuperado el 22 de octubre de 2010, de: http://ses4.sep.gob.mx/wb/ses/federales

22. Yarzábal, L. (1999). International Institute for Higher Education in LatinAmerica and the Caribbean: Bogota: IESALC/UNESCO. 